\title{
DETECTION OF SHIP TARGETS IN POLARIMETRIC SAR DATA USING 2D-PCA DATA FUSION
}

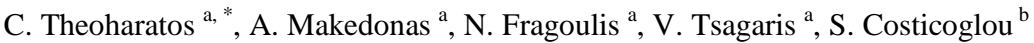 \\ ${ }^{a}$ Computer Vision Systems, IRIDA Labs S.A., Patras Science Park, Stadiou Str., Platani, 26504 Patras, Greece - (htheohar,anmack, \\ nfrag, tsagaris)@iridalabs.gr \\ ${ }^{\mathrm{b}}$ Space Hellas S.A., 312 Messogion Ave., 15341 Athens, Greece - scostic@space.gr
}

Commission VI, WG VI/4

KEY WORDS: Polarimetric data fusion, 2D-PCA, vessel detection, CFAR detector, Weibull distribution, RadarSat-2, Envisat.

\begin{abstract}
:
Data fusion has lately received a lot of attention as an effective technique for several target detection and classification applications in different remote sensing areas. In this work, a novel data fusion scheme for improving the detection accuracy of ship targets in polarimetric data is proposed, based on 2D principal components analysis (2D-PCA) technique. By constructing a fused image from different polarization channels, increased performance of ship target detection is achieved having higher true positive and lower false positive detection accuracy as compared to single channel detection performance. In addition, the use of 2D-PCA provides the ability to discriminate and classify objects and regions in the resulting image representation more effectively, with the additional advantage of being more computational efficient and requiring less time to determine the corresponding eigenvectors, compared to e.g. conventional PCA. Throughout our analysis, a constant false alarm rate (CFAR) detection model is applied to characterize the background clutter and discriminate ship targets based on the Weibull distribution and the calculation of local statistical moments for estimating the order statistics of the background clutter. Appropriate pre-processing and post-processing techniques are also introduced to the process chain, in order to boost ship discrimination and suppress false alarms caused by range focusing artifacts. Experimental results provided on a set of Envisat and RadarSat-2 images (dual and quad polarized respectively), demonstrate the advantage of the proposed data fusion scheme in terms of detection accuracy as opposed to single data ship detection and conventional PCA, in various sea conditions and resolutions. Further investigation of other data fusion techniques is currently in progress.
\end{abstract}

\section{INTRODUCTION}

Detection of ship targets is an important task in maritime and sea border surveillance, pollution control (e.g. oil spill detection), as well as vessel search and rescue in emergencies. It also stands as the first stage in a ship identification scheme. While the surveillance area and demands expand rapidly, synthetic aperture radar (SAR) becomes a powerful complementary or alternative tool to traditional surveillance resources such as Automatic Identification Systems (AIS) and Vessel Traffic Services (VTS) systems. Recently, ocean surveillance with SAR data has been researched widely. The most representative are the Canada's Ocean Monitor Workshop (OMW), the American's Alaska SAR Demo, and the series projects promoted by European Community, such as IMPAST, DECLIMS and LIMES (Margarit, 2006; LIMES, 2006).

In this context, new high resolution SAR sensors are very attractive, because they promise to improve the performance of the whole scheme. Commonly, algorithms devised to discriminate between ships and background, depend on the goodness of the background statistical characterization. The electromagnetic action between microwave and sea surface, and speckle noises make the sea clutter in SAR imagery presents statistical properties. Such images are heavily affected by the presence of the speckle, and, for this reason, many ship detection algorithms employ Constant False Alarm Rate (CFAR) algorithms (Oliver, 2004; Crisp, 2004). CFAR detectors are based on the analysis of sea clutter's statistical properties. A CFAR algorithm is an anomaly detector, which is able to find those pixels which are unlikely to be statistically characterized by the hypothesized background probability density function (pdf). There are several statistical models that are suitable to describe SAR's sea clutter characteristics such as Rayleigh, Lognormal, Weibull, K and generalized gamma distributions (Anastassopoulos, 1999; Farina, 1986; Chitroub, 2002; Farina, 1994; Erfanian, 2009). The clutter power is presented by its mean and variation values and the decision threshold that determines which pixel belongs to target is calculated based on the two known values.

During these past decades, a considerable number of CFAR detectors were presented in the literature, with different local statistics of the sea clutter (Bisceglie, 2005). Each CFAR detector requires a high computational load, depending on the background pdf, i.e. the longer the time required to estimate the pdf parameters, the higher the computational load required by the detection algorithm. As the number of SAR sensors increases and the demand for sea application in highresolution scenes grows rapidly, fast algorithms needs to be designed to deal with the mass data and to achieve wide area ocean surveillance. A conventional CFAR detector searches ship targets adaptively in the whole imagery with a sliding window, which consumes much time and cannot meet the near real-time processing requirement (Xiang, 2012).

In addition, research has recently shifted to the exploitation of polarimetric SAR data properties (Touzi, 2004; Margarit, 2006), which utilize the composite mechanisms of ships based on combinations of basic scattering procedures. The geometric properties of the polarimetric scattering behaviour can act as a good estimate for discriminating ships from sea clutter. For polarimetric data with more than two channels, 
images can be properly combined in order to exploit complementary features from different channels for improving the performance of ship target detection.

To cope with the limitations reported above, that is the need to perform effectively (with high detection accuracy) and efficiently (within near real-time limits) ship target detection in more than two channels SAR imagery, data fusion can be a very effective tool with a variety of mutli-sensor data fusion techniques developed recently. A significant amount of research has been performed on linear fusion methods, mainly for multi- and hyperspectral data (Jia, 1999; Pohl, 1998; Tyo, 2003; Tsagaris, 2005). However, little attention has been given on the utilization of polarimetric SAR data fusion approaches. For example, Hong et. al. (Hong, 2002) used multiresolution polarimetric SAR image fusion based on discrete wavelet transform (DWT) for combining spatial registered multi-polarization channels. The resulting classification map based on polarimetric feature vector presented better class separation after application of fusion processing than without fusion. In another work (Sciotti, 2001), detection techniques for exploiting the polarimetric information of SAR data were developed, in order to improve the detection capability of ships. Fusion of the polarimetric information was performed using both a decentralized and a centralized approach. Zhao et. al. (Zhao, 2008) proposed an effective polarimetric image fusion technique for improving signal to clutter contrast by utilizing the polarization scattering attributes of objects and background clutter. More recently, in their work Fei et. al. (Fei, 2012) proposed a novel Markov-chain-based CFAR detector for polarimetric data, using low-level data fusion and high-level decision fusion, which considered both correlation between neighboring pixels and pdf information in CFAR detection.

In this work, a novel data fusion scheme for improving the detection accuracy of ship targets in polarimetric data is proposed, based on 2D principal components analysis (2DPCA) technique (Yang, 2004). Compared to conventional PCA, which first rearranges the pixels in each band $A_{k}$ into column vectors $\boldsymbol{a}_{k}$, 2D-PCA operates directly on the input bands. This direct operation does not destroy (or affect) any row-to-row relationships that may exist between the pixel gray-level values. By constructing the image covariance matrix directly on $2 \mathrm{D}$ matrices, rather than $1 \mathrm{D}$ vectors, this is found much smaller. The application of 2D-PCA creates a fused image from the different polarization channels. The outcome is the achievement of increased performance of ship target detection, having higher true positive and lower false positive detection accuracy as compared to single channel detection performance. In addition, the use of 2D-PCA provides the ability to discriminate and classify objects and regions in the resulting image representation more effectively, with the additional advantage of being more computational efficient and requiring less time to determine the corresponding eigenvectors, compared to e.g. conventional PCA (Theoharatos, 2011).

Throughout our analysis, a constant false alarm rate (CFAR) detection model is applied to characterize the background clutter and discriminate ship targets based on the Weibull distribution and the calculation of local statistical moments for estimating the order statistics of the background clutter. Appropriate pre-processing and post-processing techniques are also introduced to the process chain, in order to boost ship discrimination and suppress false alarms caused by range focusing artifacts. Experimental results provided on a set of Envisat and RadarSat-2 images (dual and quad polarized respectively), demonstrate the advantage of the proposed data fusion scheme in terms of detection accuracy as opposed to single data ship detection and conventional PCA, in various sea conditions and resolutions. Further investigation of other data fusion techniques is currently in progress.

The rest of this paper is organized as follows. The SAR data description is provided in Section 2. Section 3 provides an overview of the utilized CFAR detection approach, along with the necessary parameter estimation. The utilized data fusion approach is presented in Section 4. Experimental results are provided in Section 5 and conclusions are made in Section 6 along with future research objectives.

\section{DATASET DESCRIPTION}

Two SAR scenes were used in this study. The first dataset comes from RadarSat-2 satellite mission and was acquired on May 6 2008, having fine quad polarization data $(\mathrm{HH}, \mathrm{VV}$, $\mathrm{HV}$ and VH polarizations) and resolution of $4.73 \mathrm{~m} \mathrm{x} 4.82 \mathrm{~m}$. The experiment area is located in the area of Vancouver, US, illustrated in the red rectangle in Figure 1.

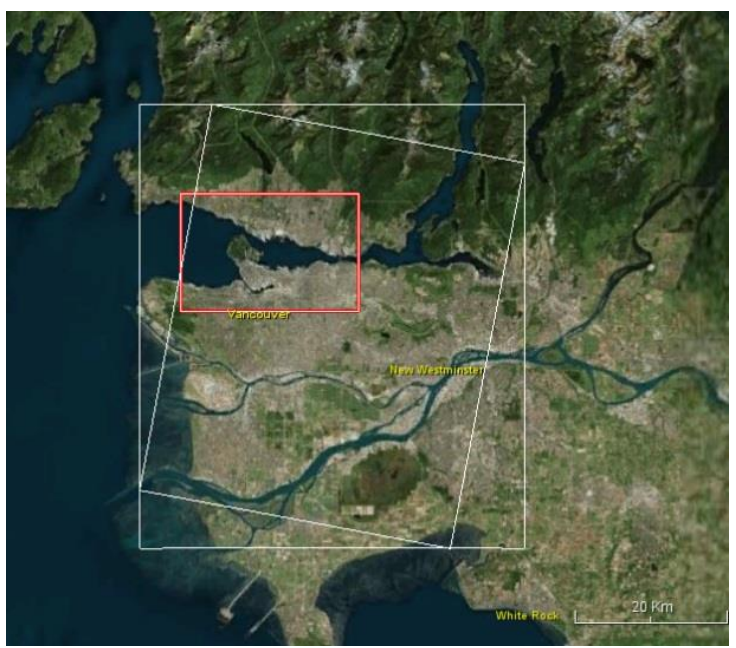

Figure 1. The quad-pol RadarSat-2 scene's test site.

The second dataset comes from Envisat satellite mission and was acquired on May 6 2008, having dual polarization data (HH and HV polarizations) and resolution of $12.5 \mathrm{~m} \mathrm{x} 12.5 \mathrm{~m}$. The experiment area is located in the area of Saronikos Golf, Greece, illustrated in the red rectangle in Figure 2.

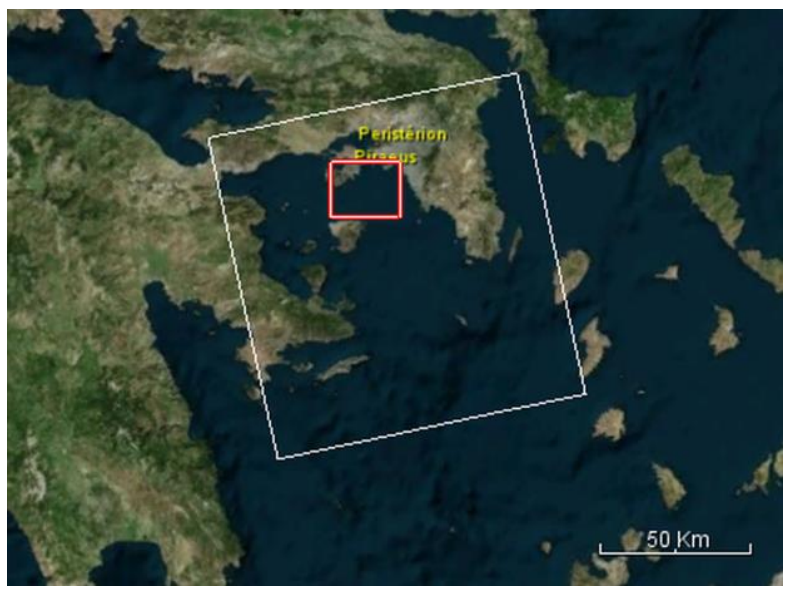

Figure 2. The dual-pol Envisat scene's test site. 


\section{CFAR DETECTION APPROACH}

In a typical CFAR detection approach, the clutter pdf is given by $p_{c}(x)$ within a test cell $Y_{c}$ corresponding to the clutter pixels only, while the target pdf is represented by $p_{t}(x)$ for target pixels within a cell $Y_{t}$ corresponding to the signalplus-clutter (Fei, 2012). Given the different statistical distributions, the target can be discriminated from the background clutter by comparing the pixel value to a threshold $T$. In this way, the probability of false alarm $P_{F A}$ is physically the area under the clutter model, while the probability of detection $P_{D}$ that indicates the area under the target model:

$$
\begin{gathered}
P_{F A}=P\left[Y_{c} \geq T\right]=\int_{T}^{\infty} p_{c}(x) d x, \\
P_{D}=P\left[Y_{t} \geq T\right]=\int_{T}^{\infty} p_{t}(x) d x
\end{gathered}
$$

In the above equations, the threshold $T$ can be defined in an adaptive way based on the clutter statistical model (e.g. mean value) of the local background, as $T=\alpha \cdot m$, with $\alpha$ being a positive empirical value.

In order to provide a reasonable fit to the amplitude statistics of the background clutter, several CFAR detectors with different local statistics have been proposed in the past. For example, in homogenous clutter environment the mean-level detectors are widely used, an example of which is cell averaging CFAR (CA-CFAR) (Gandhi, 1994). Other commonly used detectors are order statistic (OS-CFAR) (Peihong, 1996), greatest of CFAR (GO-CFAR) and smallest of CFAR (SO-CFAR) (Gandhi, 1988), optimal Weibull CFAR (OW-CFAR) (Anastassopoulos, 1995), the concept of variability index CFAR (VI-CFAR) (Smith, 1997), as well as combinations of the previous ones, for solving the problems of clutter edge and interference of neighbour target. All those types have their own pros and cons, with different potential application situations. Lately, adaptive CFAR algorithms have been proposed based on SAR data properties (Smith, 2000) or automatic censoring (Gao, 2009) for target detection.

In our analysis, a nonparametric CFAR detector was utilized, based on pairs of the ordered statistic (OS-CFAR) samples of the Weibull distribution, which has been found to provide a good fit to sea clutter.

\subsection{The Weibull distribution}

The Weibull pdf is a two-parameter distribution. The first parameter of the distribution, a shape parameter, relates to the skewness of the distribution, whereas the second parameter, a scale parameter, scales the distribution. This distribution is mathematically convenient as it allows the skewness of the distribution to be changed with a single parameter to match the characteristics of the data.

The pdf of the Weibull distribution is given by:

$$
p(x)=\frac{b}{h} \cdot \frac{x}{h} \cdot e^{-\frac{x^{b}}{a}}
$$

where

$$
\begin{aligned}
& x \geq 0 \text { and } a \geq 0, \\
& b \text { is the shape parameter and } h \text { is the scale } \\
& \text { parameter of the Weibull distribution. }
\end{aligned}
$$

The mean value is given by:

$$
E(X)=h \cdot \Gamma\left(1+\frac{1}{b}\right)
$$

where $\Gamma(\cdot)$ is the gamma function given by

$$
\Gamma(z)=\int_{0}^{\infty} t^{z-1} e^{-t} d t .
$$

In addition, the variance is given by:

$$
\operatorname{var}(X)=h^{2} \cdot\left[\Gamma\left(1+\frac{2}{b}\right)-\Gamma^{2}\left(1+\frac{2}{b}\right)\right]
$$

\subsection{Estimation of Weibull parameters}

By combining equations (4) and (5), we can estimate an expression that is independent of the parameter $h$ :

$$
C V=\frac{\sqrt{\operatorname{var}(X)}}{E(X)}=\frac{\sqrt{\Gamma\left(1+\frac{2}{b}\right)-\Gamma^{2}\left(1+\frac{2}{b}\right)}}{\Gamma\left(1+\frac{1}{b}\right)}
$$

Using eq. (6) we can calculate the value of parameter $b$ through some predefined variance values for different Weibull distributions. This can also be easily done by estimating different sets of $C V$ and $b$ values, which can be called based on an estimated look-up table.

In order to model the clutter statistics of the Weibull distribution, the scale parameter $h$ needs also to be calculated from eq. (4), given the estimated value of $h$ and the expected value of $\mathrm{x}$ within the test cell:

$$
E(X)=m=\frac{1}{N} \sum_{i=1}^{N} x_{i}
$$

In addition, the threshold value $T$ is given by:

$$
T=\alpha \cdot m=h\left[\log \left(\frac{1}{P_{F A}}\right)\right]^{2 / b}
$$

Therefore, given a value for parameter $\alpha$ (e.g. $\alpha=3$ ) and the exact value of the mean power of the clutter $m$, we can determine the threshold value $T$ and, thereafter, the scale and shape parameters $h$ and $b$ respectively.

Actually, target discrimination from the background clutter is dealt as a two-class classification problem. Given the parameter estimation model presented above and a predefined value for $P_{F A}$ (based on the uniformity or homogeneity nature of the clutter), one must determine the size of the guard and boundary cell within a sliding window, centered on the ROI. This window is typically called CFAR stencil and is shown in Fig. 3. 


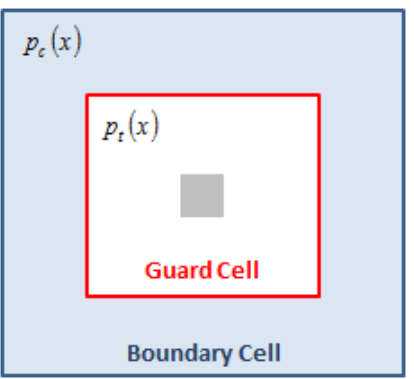

Figure 3. CFAR stencil representation.

Different types and sizes of guard and boundary cells can be utilized in the detection process. The most common choices are rectangular types of cells, the dimensions of which depend on the data resolution and the size of ship targets that need to be detected in the SAR data. If $G$ is the size of the guard cell and $B$ is the size of the boundary cell, a value of $B=3 G / 2$ is experimentally found to provide good detection results.
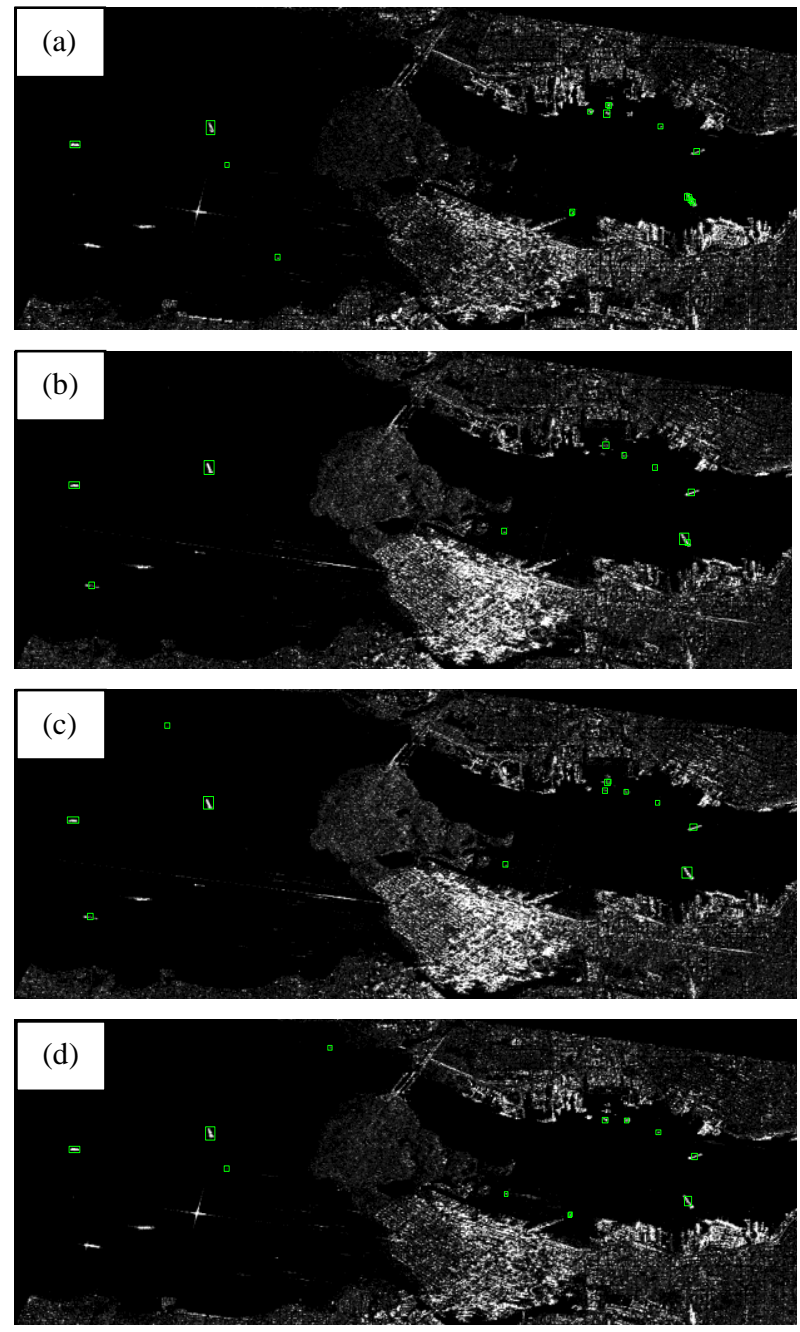

Figure 4. Detected ship targets in (a) HH, (b) HV, (c) VH and

(d) VV polarizations, for the RadarSat-2 scenes, using a

CFAR stencil of size $51 x 77$.
Figure 4 illustrates the CFAR detection results for the quadpola RadarSat-2 data, using guard and boundary sizes of values $51 \times 77$ (i.e. $G=51$ and $B=77$ ). This, however, CFAR detection approach performed on each separate polarization channel, is an extremely slow process and might not be applicable in near real-time applications.

\section{POLARIMETRIC FUSION STRATEGIES}

In order to improve target detection capabilities, execution time, and also take advantage of the different polarizations of polarimetric SAR data, data fusion strategies can be applied to combine the complementary information. Two different levels of data fusion to polarimetric CFAR detection can be applied, as shown in Figure 5:

a. A single-channel CFAR detection algorithm is applied to each polarimetric image and then the detection hits are combined using a decision fusion approach

b. The polarimetric images are first combined using a data fusion module and then a single-channel CFAR detection algorithm is applied.
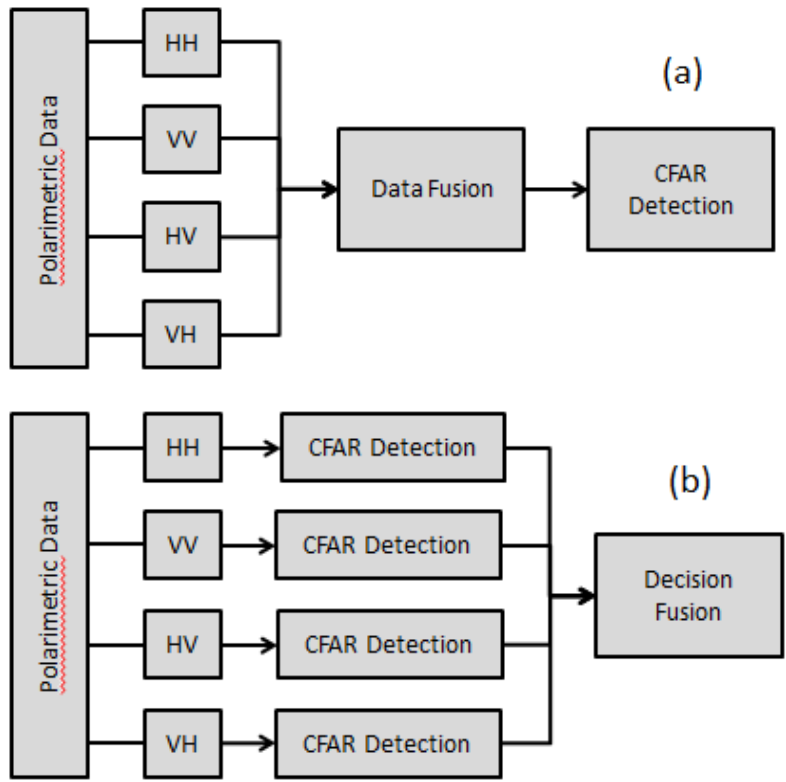

Figure 5. Polarimetric (a) data (top-diagram) and (b) decision (bottom-diagram) fusion approaches.

For both fusion approaches, the considered detection algorithm is the one described in Section 3. That is, either if CFAR detection is applied to the combined image (Fig. 5(a)) or separately to each polarimetric image (Fig. 5(b)), the modified version of the OS-CFAR using sample of the Weibull distribution is utilized in the study.

Regarding data fusion, several transformation or polarimetric decomposition techniques can be applied for improving the signal-to-noise ratio of the ship targets, as explained in the introduction. In this paper, 2D-PCA is utilized for creating a fused image from the different polarization channels, which was found to achieve increased performance of ship target detection compared to conventional PCA technique or single channel detection performance. A short description of the classical PCA and the proposed 2D-PCA is provided in the sequel. 


\subsection{PCA-based fusion of SAR data}

Principal components analysis is a widely utilized dimensionality reduction technique in remote sensing, having been considered as a novel solution for sub-band fusion processes. This is due to the fact that the information of the original multidimensional data is transformed mainly on the first few orthogonal components of the new space. The concentration of the available information on a single band results in maximization of the variance for the pixels and the features in this band. Simultaneously, information does not degenerate, since PCA is an information preserving transformation and consequently the source data can be restored. However, the use of PCA may not reveal information that appears only in a few pixels of certain bands, due to the global statistical nature of this linear transformation.

Classical PCA operates on one-dimensional data, by first transforming each input image $A_{k}$, with $k=1,2, \ldots, K$ denoting $K$ image matrices of size $M \times N$, to $M N \times 1$ size vectors and then performing eigenanalysis to each 1-D vectors. Therefore, given a set of polarimetric SAR images, PCA can be considered optimal in the sense that the first principal component will have the highest contrast and thus it can be displayed as a grayscale image with the larger percentage of visual information. This component, however, may not be suitable to form a grayscale image since the energy is not uniformly distributed and the result will not be optimal for the target detection processes, as is the case of our analysis. In addition, by rearranging the pixels in any image $A_{k}$ into a column vector $\mathbf{a}_{k}$, any row-to-row relationship that may exist between the pixel's gray-level values is lost. In order to overcome this problem, a twodimensional PCA (2D-PCA) algorithm was proposed (Yang, 2004).

\subsection{SAR fusion using 2D-PCA}

4.2.1 2D-PCA algorithm: This techniques operates directly on the input images $A_{k}$ without first converting them into column vectors $\mathbf{a}_{k}$. The algorithmic steps are explained in the sequel (Theoharatos, 2011).

First, each input image is normalized by substracting the mean image matrix $\bar{A}$ :

$$
X_{k}=A_{k}-\bar{A}
$$

where $\quad \bar{A}=\frac{1}{K} \sum_{k=1}^{K} A_{k}$

In the following step we define a $N \times N$ image covariance matrix using the formula:

$$
C=\frac{1}{K} \sum_{k=1}^{K} X_{k}^{T} X_{k}
$$

2D-PCA aims to find an optimal projection matrix $\mathbf{Y}=\left[\mathbf{y}_{1}, \mathbf{y}_{2}, \ldots, \mathbf{y}_{d}\right] \in \mathfrak{R}^{N \times d}$, with $d<K$ that minimizes the mean square reconstruction error given by:

$$
J(\mathbf{Y})=\mathbf{Y}^{T} C \mathbf{Y}
$$

Intuitively, this means that the total scatter of the projected samples is maximized after the projection of an image onto $\mathbf{Y}$. The matrix-image representation reduces the learning process of 2D-PCA to an eigencomposition problem of the covariance matrix $C$.

Let $\mathbf{y}_{\mathrm{i}}$ with $i \in\{1,2, \ldots, d\}$ define the set of $N-$ dimensional eigenvectors of the covariance matrix $C$ corresponding to the $d$ largest eigenvalues:

$$
C \mathbf{y}_{\mathrm{i}}=\lambda_{l} \mathbf{y}_{\mathrm{i}}
$$

Then, the optimal projection axis is the unitary vector that maximizes the criterion $J(\mathbf{Y})$, that is, the eigenvector of $C$ corresponding to the largest eigenvalue. Most of the times, having only one optimal projection axis is not adequate. We usually need to select a set of projection axes, $\mathbf{y}_{1}, \mathbf{y}_{2}, \ldots, \mathbf{y}_{d}$, which follow the rules coming from the orthonormal constraints and maximizing $J(\mathbf{Y})$. Actually, the optimal projection axes are the orthonormal eigenvectors of $C$ corresponding to the first $d$ largest eigenvalues.

The 2D-PCA transformation, as defined in (12), operates on the rows of the input image $A_{k}$. Other variants of this algorithm can be obtained by including a column of a diagonal based transformation.

4.2.2 2D-PCA image reconstruction: In traditional PCA the principal components can be combined with the resulted eigenvectors to reconstruct the original data. This can be also achieved in 2D-PCA to visualize the output of the fusion process (Yang, 2004).

The basic implementation that should be computed to obtain the reconstructed image $\tilde{\mathbf{A}}$ of the input image matrices $A_{k}$ is given by:

$$
\tilde{\mathbf{A}}=\sum_{k=1}^{d} \mathbf{Y}_{k} \mathbf{y}_{k}^{T} \text {, with } k=1,2, \ldots, d
$$

where $\quad \mathbf{Y}_{k}$ are the resulting principal component vectors calculated as $\mathbf{Y}_{k}=\mathbf{A} \mathbf{y}_{k}$.

Following this concept, one can choose a restricted number of dimensions (i.e. bands) $d<K$ to represent a reconstructed subimage (of the same size) of $\mathbf{A}$ as $\tilde{\mathbf{A}}_{k}=\mathbf{Y}_{k} \mathbf{y}_{k}^{T}$. Obviously, this can be obtained by summing up the first $d$ subimages in (13), which results in an approximation for $\mathbf{A}$. In our analysis, the input polarimetric SAR data is fused using the 2D-PCA algorithm, keeping the first subimage (i.e. $d=1$ ) as the output of the aforementioned technique. The application of a single CFAR detector on the fused outcome can, not only improve target detection performance in terms of effectiveness (i.e. higher true detection and lower false alarms), but also in terms of efficiency due to the decrease of the execution time equal the number of polarization channels in the SAR data.

\section{EXPERIMENTAL RESULTS}

As presented in Section 2, two SAR scenes were used in this study, a quad-pol one (HH, VV, HV and $\mathrm{VH}$ polarizations) coming from the RadarSat-2 satellite mission of resolution of 
$4.73 \mathrm{~m} \times 4.82 \mathrm{~m}$, and a dual-polarized scene $(\mathrm{HH}$ and $\mathrm{HV}$ polarizations) coming from the Envisat satellite mission of resolution $12.5 \mathrm{~m} \times 12.5 \mathrm{~m}$. All scenes have been initially preprocessed by applying ortho-rectification, radiometric calibration, land masking for separating land and water areas and speckle filtering using Lee filter (Lee, 2009) to remove speckle noise.

Figure 6 presents the CFAR detection result of the fused outcome (after performing 2D-PCA) of the RadarSat-2 data, using the same guard and boundary sizes as the ones used in Figure 4 for the individual SAR data, that is $51 \times 77$. By comparing Figures 4 and Figure 6, it can be observed that the detection result is better in the fused outcome than the one taken from each polarization channel, with almost all (except from one) ship targets having been detected.

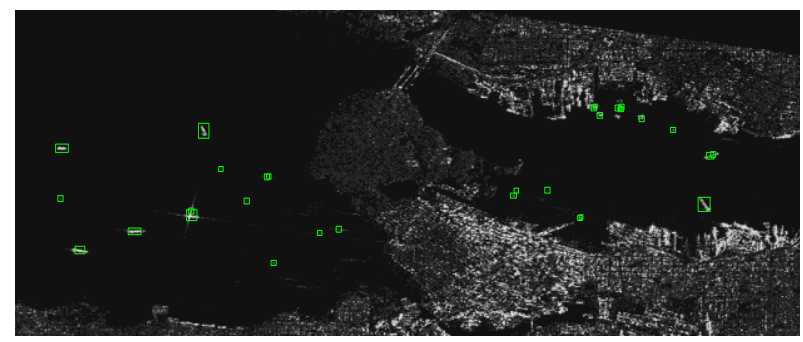

Figure 6. Detected ship targets in the 2D-PCA based fused result of the RadarSat-2 scenes, using a CFAR stencil of size $51 \times 77$.

In addition, Figure 7 illustrates the CFAR detection result of the 2D-PCA outcome of the Envisat data, using the same CFAR stencil of size $51 \times 77$.

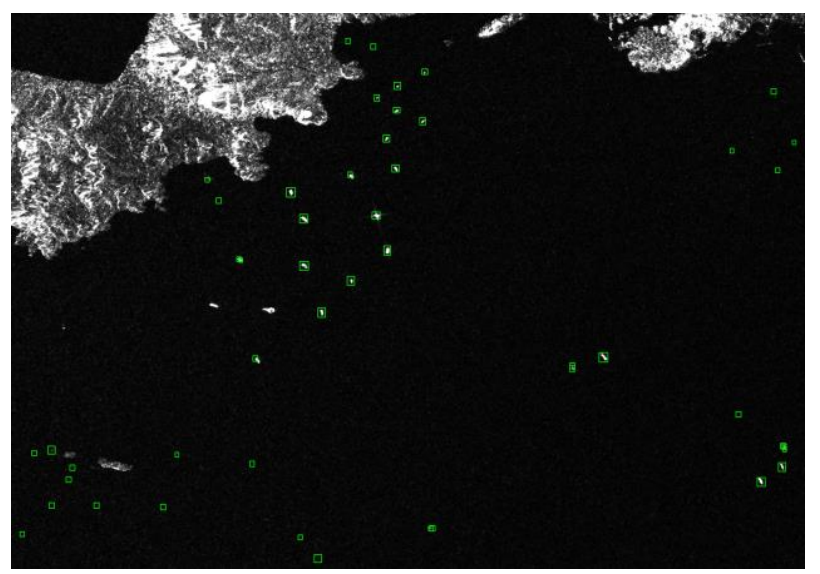

Figure 7. Detected ship targets in the 2D-PCA based fused result of the Envisat scenes, using a CFAR stencil of size $51 \times 77$.

To verify the overall detection performance, we cross checked the detected vessels with the ground truth provided by AIS ancillary data. A Figure of Merit (FoM) is used as a detection performance measurement (Foulkes, 2000). It is given by:

$$
F O M=\frac{N_{T D}}{N_{F A}+N_{G T}}
$$

where $\quad N_{T D}$ is the number of the total true detections,

$$
\begin{aligned}
& N_{F A} \text { is the number of false alarms and } \\
& N_{G T} \text { is the number of ground truth targets. }
\end{aligned}
$$

Regarding the values calculated using the formula in eq. (14), the higher the FoM numbers are, the better detection results (i.e. higher detection rate and lower false alarm rate) are taken from the application of the CFAR detector presented in Section 3. In order to calculate the FoM measure, the number of ground truth ship targets should be known apriory. These are provided from the AIS ancillary data. For the RadarSat-2 scene, the total number of ground truth targets is 20, thus $N_{G T}^{\text {RadarSat }-2}=20$, while for the Envisat data $N_{G T}{ }^{\text {Envisat }}=34$.

The application of the FoM metric to the CFAR detection results is critical, since our target is not only to detect as many targets as possible, but also to apply a technique that is able to detect as less false alarms as possible. Therefore, the detection process is a two-fold evaluation procedure.

Table 1 shows the results of detecting ships using RadarSat-2 images. The detection performance is determined by calculating the FoM at each polarization separately, as well as to the fused images generated by the application of PCA and 2D-PCA. All measurements have been taken with and

\begin{tabular}{|c|c|c|c|c|c|}
\hline \multicolumn{2}{|c|}{ mode } & $21 \times 31$ & $31 \times 47$ & $41 \times 61$ & $51 \times 77$ \\
\hline \multirow{6}{*}{ 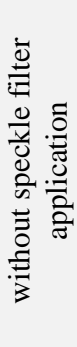 } & VV & 0,400 & 0,375 & 0,522 & 0,435 \\
\hline & VH & 0,321 & 0,258 & 0,300 & 0,478 \\
\hline & HV & 0,333 & 0,308 & 0,308 & 0,391 \\
\hline & $\mathrm{HH}$ & 0,281 & 0,176 & 0,379 & 0,312 \\
\hline & 2D-PCA & 0,391 & $\mathbf{0 , 5 0 0}$ & 0,769 & $\mathbf{0 , 5 3 1}$ \\
\hline & PCA & 0,269 & 0,292 & 0,280 & 0,304 \\
\hline \multirow{6}{*}{ 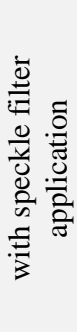 } & VV & 0,346 & 0,270 & 0,440 & 0,375 \\
\hline & VH & 0,286 & 0,300 & 0,300 & 0,360 \\
\hline & HV & 0,250 & 0,214 & 0,308 & 0,391 \\
\hline & $\mathrm{HH}$ & 0,206 & 0,212 & 0,393 & 0,312 \\
\hline & 2D-PCA & 0,346 & 0,452 & 0,600 & 0,454 \\
\hline & PCA & 0,204 & 0,160 & 0,224 & 0,219 \\
\hline
\end{tabular}
without the application of speckle filtering, which seem to be a critical pre-processing step of a target detection system.

Table 1 FoM calculation for RadarSAT-2 images.

As shown in Table 1, the use of PCA does not give good detection results in comparison to the application of 2D-PCA, providing - in most of the cases - even worse results compared to those provided by the application of the CFAR detector to each separate polarization channel. This is because the implementation of CFAR detector in the PCA produced output provides many false alarms (besides the true detections), which have a negative effect on the calculation of the FoM. The higher FoM measurements for each CFAR stencil are highlighted in bold numbers in Table 1, while the best measurement is found in red-bolded numbers. That is, the maximum value of $F o M$ is found for a CFAR stencil $41 \times 61$ pixels, without having applied a de-speckle filter procedure. 
Regarding the dual-polarization Envisat images, the ship detection performance was calculated for each one of the polarizations separately, along with the image created using the 2D-PCA. The respective FoM values are presented in Table 2. Images using simple PCA were not used, since the detection result gave too many false alarms and, therefore, the evaluation result was not evaluable. For the Envisat images, the best results were also found in the 2D-PCA based fused outcome provided using a window of $51 \times 77$, after having the data filtered to remove speckle noise. Once again, the higher FoM measurements for each CFAR stencil are highlighted in bold numbers in Table 2, while the best measurement is found in red-bolded numbers.

\begin{tabular}{|c|c|c|c|c|c|}
\hline \multicolumn{2}{|c|}{ mode } & $21 \times 31$ & $31 \times 47$ & $41 \times 61$ & $51 \times 77$ \\
\hline \multirow{3}{*}{ 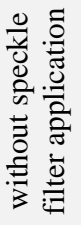 } & $\mathrm{HV}$ & 0,527 & 0,620 & 0,520 & 0,571 \\
\hline & $\mathrm{HH}$ & 0,527 & 0,633 & 0,620 & 0,646 \\
\hline & 2D-PCA & $\mathbf{0 , 5 3 7}$ & $\mathbf{0 , 6 6 7}$ & 0,630 & 0,652 \\
\hline \multirow{3}{*}{ 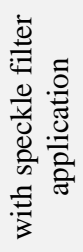 } & $\mathrm{HV}$ & 0,527 & 0,646 & 0,630 & 0,667 \\
\hline & $\mathrm{HH}$ & 0,537 & 0,633 & 0,520 & 0,617 \\
\hline & 2D-PCA & $\mathbf{0 , 5 3 7}$ & 0,667 & 0,652 & 0,727 \\
\hline
\end{tabular}

Table 2 FoM calculation for Envisat images.

Finally, concerning processing time, the application of the CFAR detector on a single polarization channels requires approximately 304.6 secs for a CFAR stencil of size $21 \times 31$ and approximately 402.5 secs for a CFAR stencil of size $51 \times 77$, running on a standard Wintel 64-bit PC, having 8GB RAM memory and an Intel Core i5 CPU processor @ 3.10GHz. It is apparent that, the application of CFAR on each separate SAR channel significantly limits the target detection capabilities, making it unmanageable for near real-time applications.

\section{CONCLUSIONS}

In this paper, a novel data fusion scheme for improving the effectiveness and efficiency of ship target detection in polarimetric data is proposed, based on $2 \mathrm{D}$ principal components analysis (2D-PCA) technique. The application of the 2D-PCA algorithm increases the detection capabilities of a classical CFAR detector, while reducing false alarm detection. In addition, it significantly reduced processing time by a factor equal to the number of incoming polarization channels. Moreover, the use of 2D-PCA provides the ability to discriminate and classify objects and regions in the resulting image representation more effectively, with the additional advantage of being more computational efficient and requiring less time to determine the corresponding eigenvectors, compared to classical approaches like the conventional PCA.

Throughout our analysis, a constant false alarm rate (CFAR) detection model is applied to characterize the background clutter and discriminate ship targets based on the Weibull distribution and the calculation of local statistical moments for estimating the order statistics of the background clutter. Appropriate pre-processing and post-processing techniques are also introduced to the process chain, in order to boost ship discrimination and suppress false alarms caused by range focusing artifacts. Experimental results provided on a set of Envisat and RadarSat-2 images (dual and quad polarized respectively), demonstrate the advantage of the proposed data fusion scheme in terms of detection accuracy as opposed to single data ship detection and conventional PCA, in various sea conditions and resolutions. Further investigation of other data fusion techniques is currently in progress.

\section{ACKNOWLEDGEMENTS}

This work was supported in part by the Hellenic General Secretariat for Research and Technology (GSRT), in the frame of project "ACRITAS", carried out within the Hellenic Space Technologies and Applications cluster (si-Cluster).

\section{REFERENCES}

Anastassopoulos, V., Lampropoulos, G.A., 1995. Optimal CFAR detection in Weibull clutter, IEEE Trans. on Aerospace and Electronic Systems. 31(1), pp. 52-64.

Anastassopoulos, V., Lampropoulos, G.A., Drosopoulos, A., Rey, M., 1999. High resolution radar clutter statistics. IEEE Trans. Aerospace and Electronics Systems, 35(1), pp. 43-60.

Bisceglie, M. Di, Galdi, C., 2005. CFAR detection of extended objects in high-resolution SAR images, IEEE Trans. on Geoscience and Remote Sensing, 43(4), pp. 833843.

Chitroub, S., Houacine, A., Sansal, B., 2002. Statistical characterization and modelling of SAR images, Signal Processing, 82(1), pp. 69-92.

Crisp, D.J., 2004. The State-of-the-Art in Ship Detection in Synthetic Aperture Radar Imagery. Intelligence, Surveillance and Reconnaissance Division Information Sciences Laboratory, DSTO-RR-0272.

Erfanian, S., Vakili, V.T., 2009. Introducing excision switching-CFAR in $\mathrm{K}$ distributed sea clutter, Signal Processing, 89(6), pp.1023-1031.

Farina, A., Lombardo, P., 1994. Modelling of a mixture of Kdistributed and Gaussian clutter for coherent radar detection, Electronics Letters, 30(6), pp. 520-521.

Farina, A., Russo, A., Studer, F.A., 1986. Coherent radar detection in log-normal clutter, In: IEE Proceedings in Communications, Radar and Signal Processing, 133(1), pp.39-54.

Fei, C., Liu, T., Lampropoulos, G.A., Anastassopoulos, V., 2012. Markov Chain CFAR Detection for Polarimetric Data Using Data Fusion, IEEE Trans. on Geoscience and Remote Sensing, 50(2), pp. 397-408.

Foulkes, S.B., Dooth, D.M., 2000. Ship detection in ERS and radarsat imagery using a self-organising Kohonen neural network, Proceedings of Nova Scotia Conference on Ship Detection in Coastal Waters, Canada.

Gandhi, P.P., Kassam, S.A., 1988. Analysis of CFAR processors in nonhomogenous background, IEEE Transaction on Aerospace and Electronic System, pp. 427445 . 
Gandhi, P.P., Kassam, S.A., 1994. Optimality of the cell averaging CFAR detector, IEEE Trans. on Information Theory, 40(4), pp. 1226-1228.

Gao, G., Liu, L., Zhao, L., Shi, G., Kuang, G., 2009. An adaptive and fast CFAR algorithm based on automatic censoring for target detection in high-resolution SAR images, IEEE Trans. on Geoscience and Remote Sensing, 47(6), pp. 1685-1697.

Hong, S., Moon, W.M., Paik, H.-Y., Choi, G.-H., 2002. Data Fusion of multiple Polarimetric SAR images using Discrete Wavelet Transform (DWT). In: Geoscience and Remote Sensing Symposium 2002 (IGARSS '02), vol. 6, pp. 33233325 .

Jia, X., Richards, J.A., 1999. Segmented principal components transformation for efficient hyperspectral remote sensing image display and classification, IEEE Trans. on Geoscience and Remote Sensing, 37(1), pp. 538-542.

Lee, J.S., Jurkevich, L., Dewaele, P., Wambacq, P., Oosterlinck, 2009. A., Speckle filtering of synthetic aperture radar images: A review, Remote Sensing Reviews, pp. 313340 .

Limes 2006. Land and Sea Integrated Monitoring for European Security (LIMES) project web page. In: Proc. 6th Framework Programme, Brussels, Belgium, 2006. Available online: http://www.fp6-limes.eu/uploads/docs/LIMESD8000.2-FRS-1\%200.pdf.

Margarit, G. Mallorqui, J.J., Rius, J.M., Sanz-Marcos, J., 2006. On the usage of GRECOSAR, an orbital polarimetric SAR simulator of complex targets, to vessel classification studies. IEEE Trans. on Geoscience and Remote Sensing, 44(12), pp. 3517-3526.

Oliver, C., Quegan, S., 2004. Understanding synthetic aperture radar images. Cap. 10, SciTech Publishing Inc., Raleigh (NC).

Peihong, R., et al., 1996. The research on the detection performance of OS-CFAR and its modified methods, In: Proc. of CIE International Conference on Radar 1996, pp. 422-425.

Pohl, C., Genderen, J.L., 1998. Multisensor image fusion in remote sensing: concepts, methods, and applications, International Journal of Remote Sensing, 19(5), pp. 823-845.

Sciotti, M., Pastina, D., Lombardo, P., 2001. Polarimetric detectors of extended targets for ship detection in SAR images. In: Geoscience and Remote Sensing Symposium 2001 (IGARSS '01), vol. 7, pp.3132-3134.

Smith, M.E., Varshney, P.K., 1997. VI-CFAR: a novel CFAR algorithm based on data variability, In: IEEE International Radar Conference, pp. 263-268.

Smith, M.E., Varshney, P.K., 2000. Intelligent CFAR processor based on data variability, IEEE Trans. on Aerospace and Electronics Systems, 36(3), pp. 837-847.

Theoharatos, C., Tsagaris, V., Fragoulis, N., Economou, G., 2011. Hyperspectral image fusion using 2-D principal component analysis, In: 2nd International Conference on Space Technology (ICST), Athens, Greece.
Touzi, R., Raney, R.K., Charbonneau, F., 2004. On the use of permanent symmetric scatterers for ship characterization, IEEE Trans. on Geoscience and Remote Sensing, 42(10), pp. 2039-2045.

Tsagaris, V., Anastassopoulos, V., Lampropoulos, G.A., 2005. Fusion of hyperspectral data using segmented PCT for color representation and classification, IEEE Trans. on Geoscience and Remote Sensing, 43(10), pp. 2365-2375.

Tyo, J., Konsolakis, A., Diersen, D., Olsen, R.C., 2003. Principal-components-based display strategy for spectral imagery, IEEE Trans. on Geoscience and Remote Sensing, 41(3), pp. 708-718.

Xiang, X., Kefeng, J., Huanxin, Z., Jixiang, S., 2012. A fast ship detection algorithm in SAR imagery for wide area ocean surveillance, In: Proc. IEEE Radar Conference 2012 (RADAR 2012), pp. 570-574.

Yang, J., Zhang, D., Frangi, A.F., Yang, J.-Y., 2004. Twodimensional PCA: a new approach to appearance-based face representation and recognition, IEEE Trans. on Pattern Analysis and Machine Intelligence, 26(1), pp. 131-137.

Zhao, Y.-Q., Gong, P., Pan, Q., 2008. Object detection by spectropolarimeteric imagery fusion, IEEE Trans. on Geoscience and Remote Sensing, 46(10), pp. 3337-3345. 